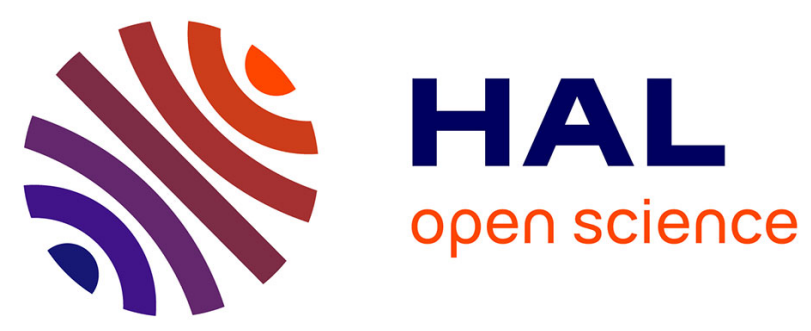

\title{
Nonlinear modeling and estimation of force in a piezoelectric cantilever.
}

\author{
Micky Rakotondrabe, Yassine Haddab, Philippe Lutz
}

\section{To cite this version:}

Micky Rakotondrabe, Yassine Haddab, Philippe Lutz. Nonlinear modeling and estimation of force in a piezoelectric cantilever.. IEEE/ASME International Conference on Advanced Intelligent Mechatronics, AIM'07., Sep 2007, ETH Zürich, Switzerland. sur CD ROM - 6 p. hal-00172927

\section{HAL Id: hal-00172927 \\ https://hal.science/hal-00172927}

Submitted on 18 Sep 2007

HAL is a multi-disciplinary open access archive for the deposit and dissemination of scientific research documents, whether they are published or not. The documents may come from teaching and research institutions in France or abroad, or from public or private research centers.
L'archive ouverte pluridisciplinaire HAL, est destinée au dépôt et à la diffusion de documents scientifiques de niveau recherche, publiés ou non, émanant des établissements d'enseignement et de recherche français ou étrangers, des laboratoires publics ou privés. 


\title{
Nonlinear modeling and estimation of force in a piezoelectric cantilever
}

\author{
Micky Rakotondrabe, Yassine Haddab and Philippe Lutz
}

\begin{abstract}
This paper deals with the estimation of force in a piezoelectric cantilever. The nonlinearities, i.e. hysteresis and creep, are taken into account. A nonlinear model relating the force, the voltage and the resultant deflection is first developped. The proposed estimator has been experimented and compared with the classical linear estimator. The results confirm the interest of the proposed method in term of accuracy.

Index Terms - Modeling, estimation, force, nonlinear, piezoelectric cantilever.
\end{abstract}

\section{INTRODUCTION}

In micromanipulation, a high positioning accuracy is required. In addition, phenomena neglected in macromanipulation becomes paramount when the sizes of the manipulated parts are very small. These phenomena, called scale effects, are mainly the adhesion forces [1]. In order to achieve successfully a micromanipulation task, the design of the micromanipulators and the microrobots must takes into account the characteristics of the microworld: high accuracy and adhesion forces. For that, instead of using joints, one uses active materials as microactuators. Because of their good resolution, good accuracy and high speed, piezoelectric materials still stay loyal systems for microactuators. One of the applications of piezoelectric materials is the microgripper (Fig. 1) [2][3]. Here, the piezoelectric microgripper is made up of two piezoelectric cantilevers.

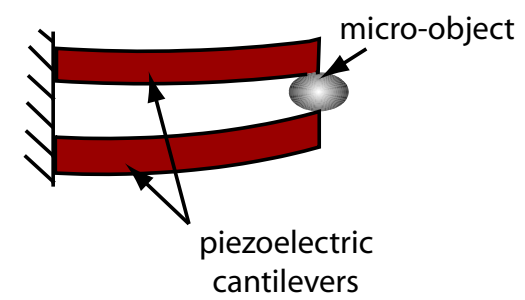

Fig. 1. Two piezoelectric cantilevers used as a microgripper.

In order to avoid the destruction of the parts during a micromanipulation task, in particular biological or optical parts, it is crucial to take into account the

Laboratoire d'Automatique de Besançon

LAB CNRS UMR6596

ENSMM - Université de Franche-Comté

24, rue Alain Savary

25000 Besançon - France

\{mrakoton, yhaddab, plutz\}@ens $2 m$.fr handling force. However, having force sensors adapted for microgrippers is very difficult because of the necessary accuracy and sizes. Then, instead of using sensors, one of the possibilities is the use of a force estimator. In [4], a linear force estimator has been proposed. It is based on a linear dynamic model between the applied voltage, the applied force and the resultant deflexion on a piezoelectric cantilever. However, when the volatge becomes large, typically higher than $15 \%$ of the maximal field strength [5], the linear relation between the deflection and the voltage is not applicable anymore and hysteresis and creep phenomena arise. This paper deals with a modeling and a force estimator in a piezoelectric cantilever taking into account the nonlinearities.

In the second section, the relation between the applied voltage and the resultant deflection is developped. The relation between the applied force and the corresponding deflection is developped in the third section. The complete model, i.e. the nonlinear model relating the voltage, the force and the deflection, is given in section five. The last section presents the nonlinear force estimator and the experiments.

\section{Modeling of the VOltaGe-DEFLECTION TRANSFER}

When applying an electrical excitation $U$ to a piezoelectric cantilever or a mechanical excitation $F$ at its tip (Fig. 2), the classical working relation is [6]:

$$
\delta=\left(d_{p} \cdot U+s_{p} \cdot F\right) \cdot D(s)
$$

where $\delta$ is the obtained deflection, $d_{p}$ and $s_{p}$ are respectively a piezoelectric constant and an elastic constant associated to the whole cantilever. The dynamic part is represented by $D(s)$, with $s$ the Laplace variable.

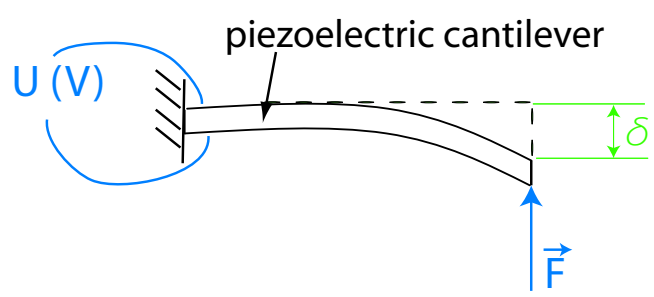

Fig. 2. Bending of a piezoelectric cantilever. 
When the applied voltage $U$ becomes large, hysteresis and creep effect arise (Fig. 3). The creep is defined as a drift of the deflection after the transient part (Fig. 3b). In this section, we describe the relation between the deflection and the voltage taking into account these nonlinearities. For that, the force is considered to be null. Let the expression $\delta=d_{p} . U$ be replaced by $\delta=\Gamma($.$) ,$ where $\Gamma($.$) is an operator incorporating the hysteresis$ and the creep. The notation '(.)' means that the operator depends on the present and on the past of the voltage. (a)

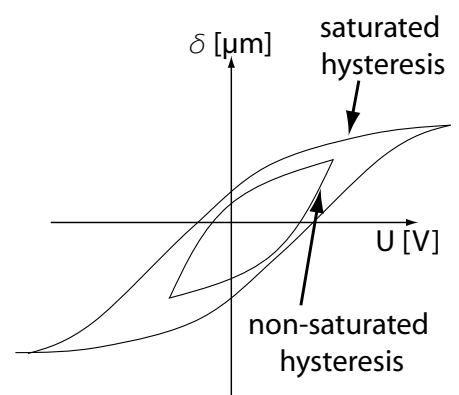

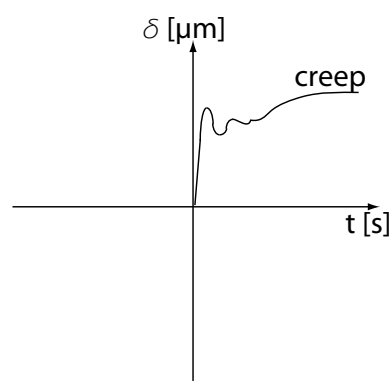

(b)
Fig. 3. a: hysteresis phenomenon. b: creep phenomenon.

For all the experiments, we use a piezoelectric unimorph cantilever made up of a PZT layer (Lead Zirconate Titanate) and a metallic layer (Copper). Its sizes are $15 \mathrm{~mm} \times 2 \mathrm{~mm} \times 0.3 \mathrm{~mm}$ (length, width and total thickness) (Fig. 4).

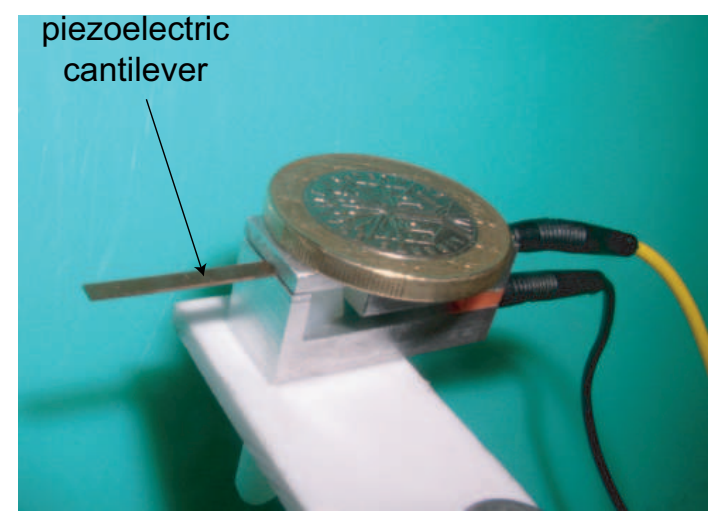

Fig. 4. Photography of the piezoelectric cantilever.

\section{A. Systemic scheme}

First, let us study the hysteresis. Using a harmonic analysis, it is easy to show that the hysteresis shape of a piezoelectric cantilever depends on the applied frequency. Such an hysteresis is called dynamical hysteresis (or ratedependent hysteresis) [7]. However, our previous work [8] has shown that for piezoelectric cantilevers, the dynamical hysteresis $H_{d}($.$) is equivalent to a statical hysteresis$
$H_{i}($.$) in serie with a linear dynamical part D(s)$ (Fig. 5). A statical hysteresis (or rate-independant hysteresis) has a constant shape whatever the frequency is.

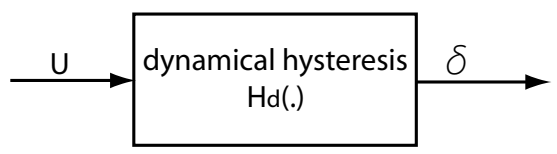

(a)

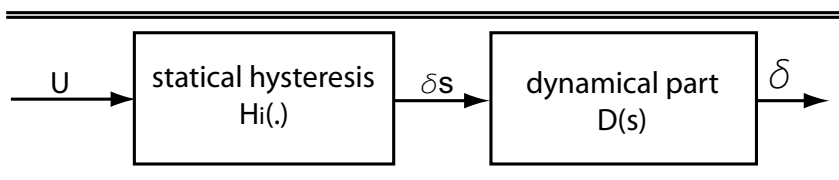

(b)

Fig. 5. The dynamical hysteresis $H_{d}($.$) is equivalent to a statical$ hysteresis $H_{i}($.$) in serie with a dynamical part D(s)$ [8].

Thus:

$$
H_{d}(.)=H_{i}(.) \cdot D(s)
$$

According to this equation, the hysteresis only affects the statical gain of the voltage-deflection transfer.

Now, let us apply a step voltage to a piezoelectric cantilever. The creep phenomenon happens just after the transient part (Fig. 6-a). It can be considered as an additional behavior happening when the steady-state is reached. However, the response time of the transient part is very small in comparison with the response time of the creep: less than $80 \mathrm{~ms}$ for the former and more than $2 \mathrm{~min}$ for the latter. So, for the simplification, we assume that the creep starts in the same time than the transient part (Fig. 6-b).
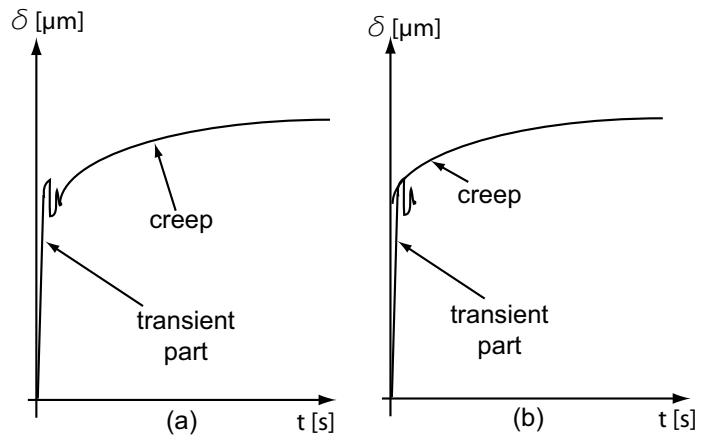

Fig. 6. a: the creep begins just after the transient part. b: the duration of the transient part can be neglected.

Let $C_{r}(s)$ represent the model of the creep phenomenon. From the precedent remarks, we can deduct the systemic scheme relating the voltage and the deflection (Fig. 7). The corresponding formulation is as follow: 


$$
\delta=C_{r}(s) \cdot U+H_{i}(.) \cdot D(s)
$$

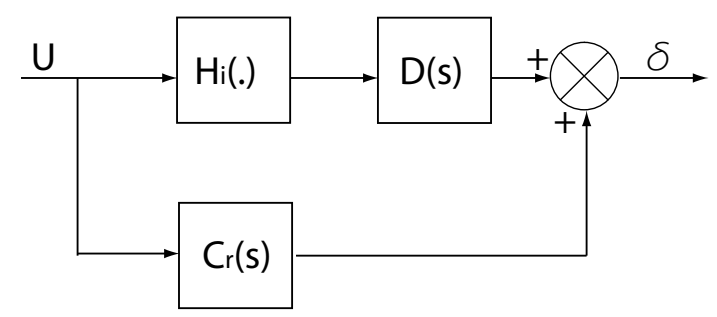

Fig. 7. Relation between the deflection and the voltage.

Now, let us determine the hysteresis model $H_{i}($.$) , the$ dynamical part $D(s)$ and the creep model $C_{r}(s)$.

\section{B. Model of the statical hysteresis $H_{i}($.}

The hysteresis of a piezoelectric cantilever have been subjected to various models. The one which offers a simplicity in term of mathematical computing is based on the Bouc-Wen model [9][10]. It is a set of nonlinear differential equations. For piezoelectric cantilever, the model is given by the following set of equations [11][12]:

$$
\begin{gathered}
\delta=d_{p} \cdot U-z \\
\dot{z}=A^{b w} \cdot \dot{U}-B^{b w} \cdot|\dot{U}| \cdot z-\Gamma^{b w} \cdot \dot{U} \cdot|z|
\end{gathered}
$$

where $d_{p}$ is the previous piezoelectric constant and $z$ is an internal state (restoring force) introducing the hysteresis. The coefficient $A^{b w}$ controls the amplitude of the restoring force while the coefficients $B^{b w}$ and $\Gamma^{b w}$ control the shape of the hysteresis. The parameters identification is performed using a low frequency sine voltage in order to ensure a working domain where the model is statical. We have:

$$
\left\{\begin{array}{l}
d_{p}=1.0773 \times 10^{-6} \mathrm{~m} / \mathrm{V} \\
A^{b w}=6.064810 \times 10^{-7} \mathrm{~m} / \mathrm{V} \\
B^{b w}=0.00833 \mathrm{~V}^{-1} \\
\Gamma^{b w}=0.00833 V^{-1}
\end{array}\right.
$$

\section{Model of the dynamic part $D(s)$}

The dynamic model $D(s)$ can be identified by using the transient part of one step response (Fig. 8). This is acceptable because the hysteresis only affects the statical part while the creep happens after the transient part. A second order model is assumed to be sufficient [4][12]. Using the ARMAX method, we obtain:

$$
D(s)=\frac{-4.814 \times 10^{-3} \cdot \mathrm{s}^{2}+919 \cdot \mathrm{s}+2 \times 10^{7}}{s^{2}+459 \cdot s+2 \times 10^{7}}
$$

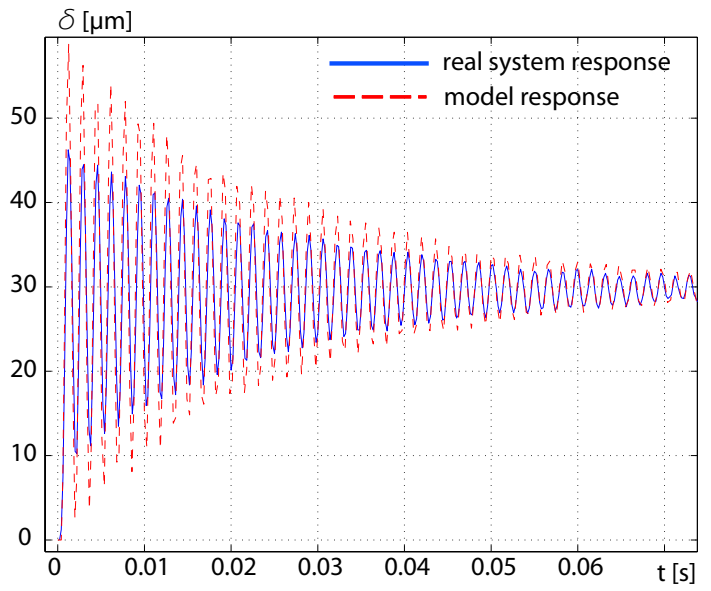

Fig. 8. Step response of the piezoelectric cantilever.

\section{Model of the creep $C_{r}(s)$}

The creep can be considered as the result of a dynamical linear system [13]. In general, a first order linear system can largely model it. To identify the model, we again apply a step to the piezoelectric cantilever. After applying the ARMAX-method, the obtained model is:

$$
C_{r}(s)=0.068 \cdot\left(\frac{225 \times 10^{-6} \cdot \mathrm{s}+1}{15.56 \cdot \mathrm{s}+1}\right)
$$

The Fig. 9 represents the step response of the real system (creep part) and of the identified model $C_{r}(s)$.

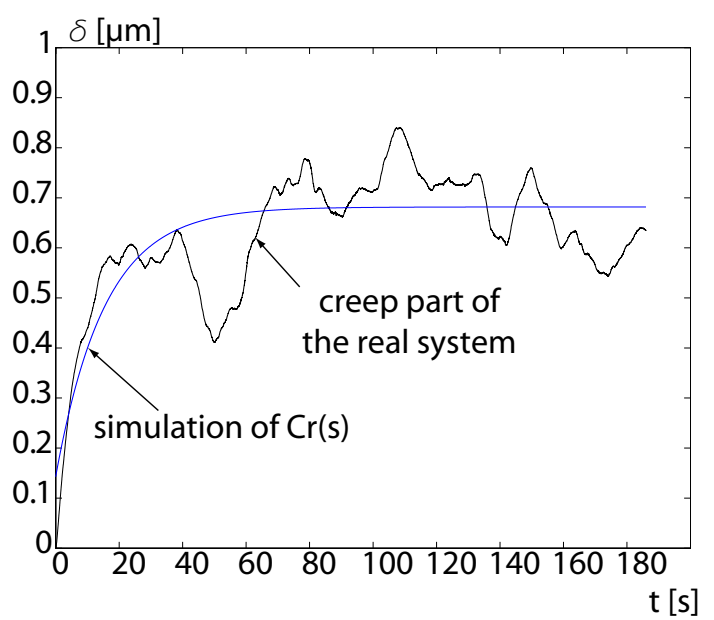

Fig. 9. Simulated and measured deflection.

\section{Modeling THE FORCE-DEFLECTION TRANSFER}

The previous section has given the nonlinear relation between the voltage $U$ and the resultant deflection $\delta$. In this section, we consider the relation between the applied force $F$ and the deflection.

In [14], it has been stated that the relation between the deflection, the force and the voltage, when the electromechanical transfer is nonlinear, is as follow: 


$$
\delta=s_{p} \cdot F+\Gamma(U)
$$

However, it is a priori unkown if the elastic (forcedeflection) relation is linear or nonlinear. Thus, the two first parts of this section is about the verification of the presence or the absence of hysteresis and creep. For all force-deflection characterizations and identifications, the input voltage is null $(U=0)$ and the electrodes are shortcircuited in order to make the voltage null.

\section{A. Hysteresis verification}

This experiment consists in putting a weight at the tip of the cantilever, removing it and putting it back again. The principle of the experiment is presented in the Fig. 10-a while the results in the Fig. 10-b. As shown, the deflection always takes either $0 \mu m$ or $51 \mu m$, each one corresponding respectively to null mass and hung mass. As there is no residual deflection, we confirm that there is no mechanical hysteresis inside the working domain $([-50 \mu m, 50 \mu m])$.

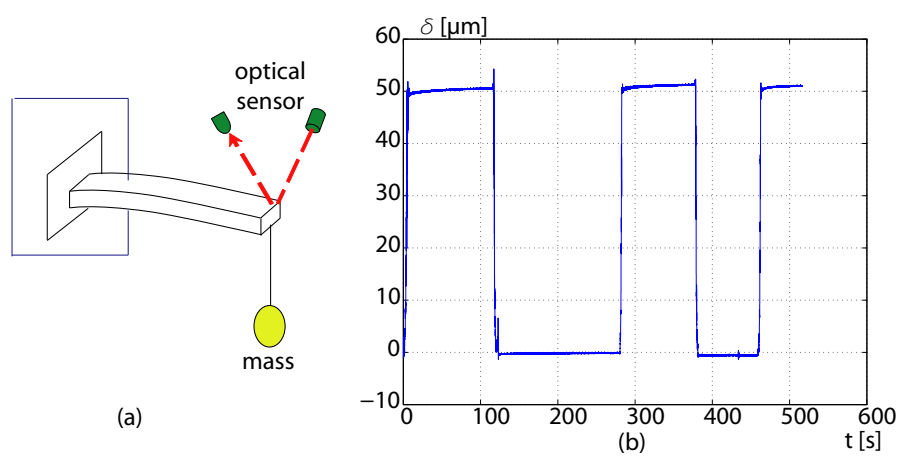

Fig. 10. Hysteresis verification.

\section{B. Creep verification}

In this experiment, the mass is hung at the tip of the cantilever during an extended period of time (up to $3 \mathrm{~min}$ ). As shown in the results (Fig. 11), the deflection still stays constant after the transient part. We conclude that there is no creep between the force and the deflection.

\section{Statical characteristic}

As there is no hysteresis, the statical gain (elastic coefficient) $s_{p}$ of the piezoelectric cantilever is constant. To identify it, a known mass $m$ is hung at the tip of the cantilever. We obtain:

$$
s_{p}=\frac{\delta}{m \cdot g}=1.931 \mu m / m N
$$

with $g \approx 10 \mathrm{~N} / \mathrm{kg}$ is the terrestrial acceleration.

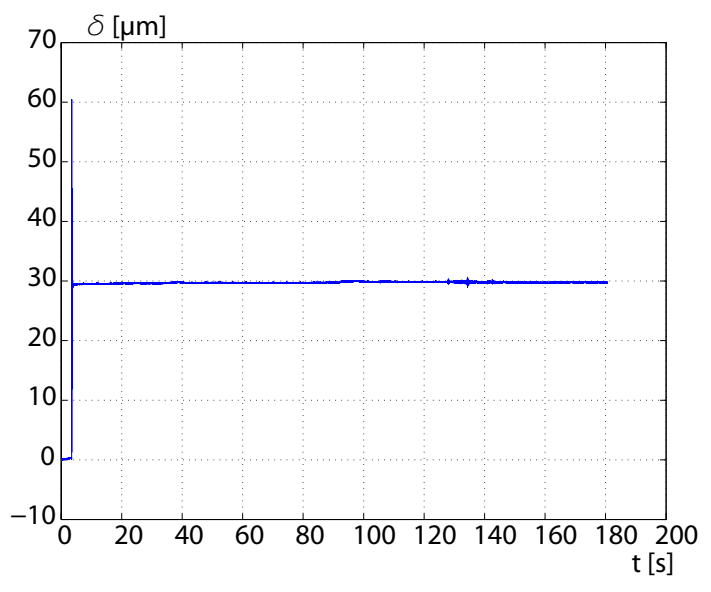

Fig. 11. Creep verification.

\section{Dynamical characteristic}

To identify the dynamical part, we use a canonical force input. As a mechanical step is difficult to apply, we choose a mechanical impulse by applying a shock to the cantilever. The corresponding response is next compared with the impulse response of $D(s)$ expressed by the equation (7). The results show that the transient part of the force-deflection transfer is almost similar to the transient part of the voltage-deflection transfer $D(s)$ (Fig. 12). Using a higher model order would give a better correlation between the curves.

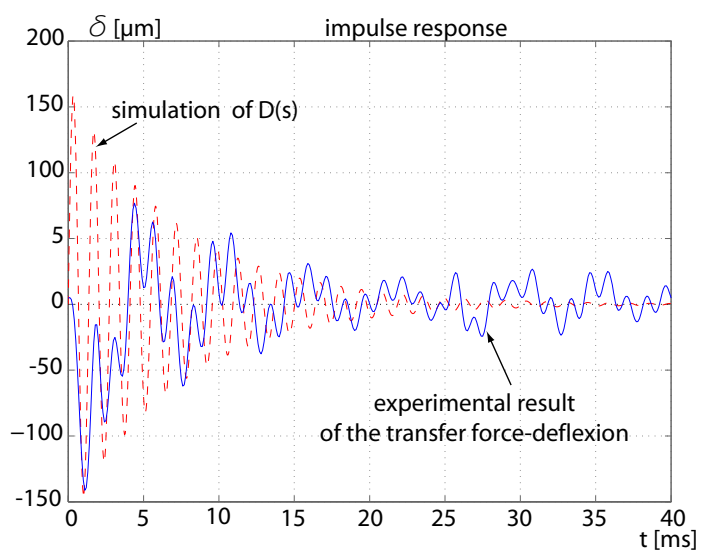

Fig. 12. Comparison between the transient part of the forcedeflection transfer and the voltage-deflection transfer.

\section{Complete nonlinear model Relating the VOLTAGE, THE FORCE AND THE DEFLECTION}

Combining the different precedent remarks, the complete nonlinear model has the following form:

$$
\delta=s_{p} \cdot F \cdot D(s)+C_{r}(s) \cdot U+H_{i}(.) \cdot D(s)
$$

Replacing the statical hysteresis operator $H_{i}($.$) by the$ equations (4) and (5), we have the final model: 


$$
\left\{\begin{array}{l}
\delta=\left(s_{p} \cdot F+d_{p} \cdot U-z\right) \cdot D(s)+U \cdot C_{r}(s) \\
\dot{z}=A^{b w} \cdot \dot{U}-B^{b w} \cdot|\dot{U}| \cdot z-\Gamma^{b w} \cdot \dot{U} \cdot|z|
\end{array}\right.
$$

\section{Estimation OF THE FORCE}

\section{A. Equation of estimation}

From the set of equations (12), we infer the force:

$$
\left\{\begin{array}{l}
F=\frac{1}{s_{p}} \cdot\left[\left(\delta-U \cdot C_{r}(s)\right) \cdot D(s)^{-1}-d_{p} \cdot U+z\right] \\
\dot{z}=A^{b w} \cdot \dot{U}-B^{b w} \cdot|\dot{U}| \cdot z-\Gamma^{b w} \cdot \dot{U} \cdot|z|
\end{array}\right.
$$

Where $D(s)^{-1}$ must be causal. Without any loss of generality, we suppose that we work in statical mode so that $D(s)=1$. In fact, the main origins of the imprecision in the force estimation are the creep and the hysteresis. Let $\widehat{F}$ be the estimated force. The set of equations for the force estimation is finally:

$$
\left\{\begin{array}{l}
\widehat{F}=\frac{1}{s_{p}} \cdot\left[\delta-U \cdot C_{r}(s)-d_{p} \cdot U+h\right] \\
\dot{z}=A^{b w} \cdot \dot{U}-B^{b w} \cdot|\dot{U}| \cdot z-\Gamma^{b w} \cdot \dot{U} \cdot|z|
\end{array}\right.
$$

The corresponding bloc-scheme is presented in the Fig. 13.

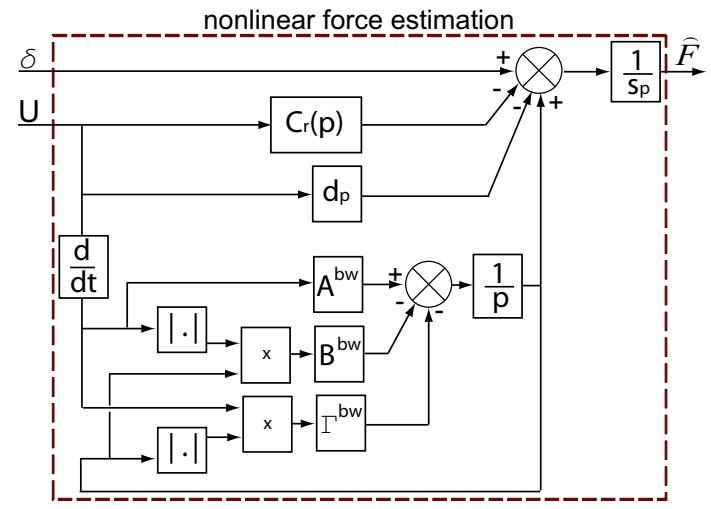

Fig. 13. Bloc-scheme of the force estimation.

\section{B. Experiments}

The proposed force estimator has been implemented inside a LabView software in a Windows-XP environment. To measure the deflection, we use an optical sensor (Keyence) with $10 \mathrm{~nm}$ resolution and 40 $\mathrm{nm}$ accuracy. The setup principle is presented in the Fig. 14. The experiments procedure is as follow:
- the force, to be estimated, applied at the tip of the cantilever and the voltage are initially null,

- at $t=3 \mathrm{~s}$, we apply a voltage $U=-30 \mathrm{~V}$,

- at $t=13 \mathrm{~s}$, a force is applied. Without loss of generality, we choose $F=20 \mathrm{mN}$ which corresponds to the manipulation force of most applications.

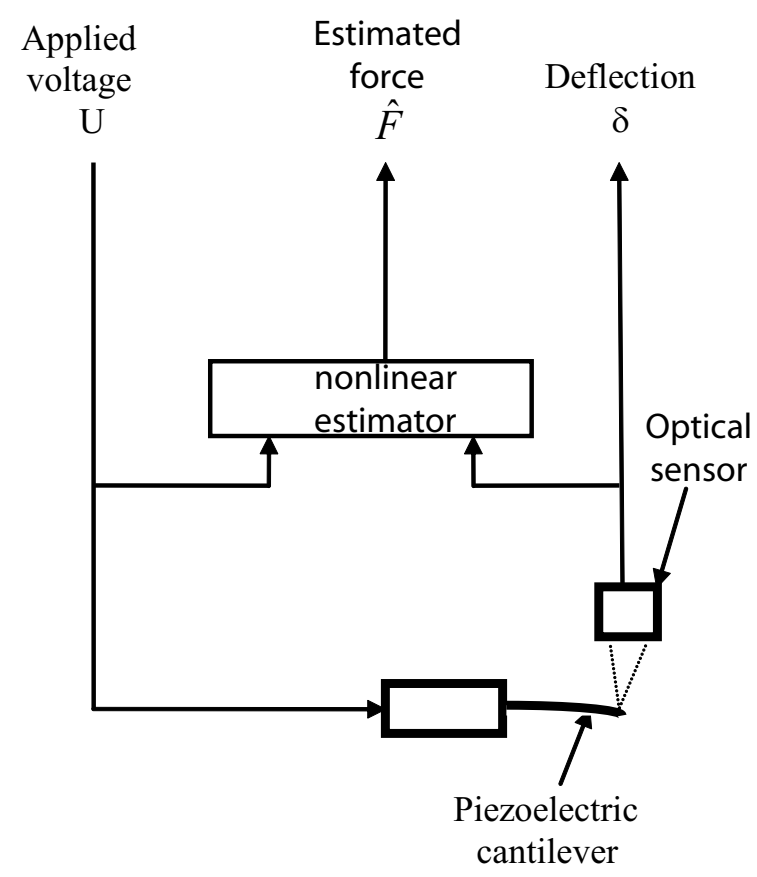

Fig. 14. Principle of the experiments.

In order to evaluate the advantages of the proposed estimator, it has been compared with the linear estimator proposed in [4] and based on the equation (1).

The voltage is presented in the Fig. 15-a while the estimated force in the Fig. 15-b.

When the step voltage is applyed $(t=3 s)$, a high pick can be observed with the nonlinear estimator. This is due to the derivation $\dot{U}$ inside the equations. Between $t=3 \mathrm{~s}$ and $t=13 \mathrm{~s}$ where the real force $F=0 \mathrm{mN}$, because of the hysteresis in the deflection, the linear estimator gives an offset about $1.5 \mathrm{mN}$. In addition, it presents a drift due to the creep. On the other hand, the nonlinear estimator minimizes these effects. When the real force $F=20 \mathrm{mN}$ $(t \geq 13 s)$, the linear estimator gives an accuracy about $1.4 \mathrm{mN}$ while the nonlinear estimator better than $0.5 \mathrm{mN}$. From these results, we deduce that the relative accuracy is about $0.05 \mathrm{mN} / \mathrm{V}$ for the linear estimator and about $0.017 \mathrm{mN} / \mathrm{V}$ for the nonlinear one.

\section{CONClusion}

In micromanipulation, the recognition of the handling force is necessary with a view to avoid the destruction of 

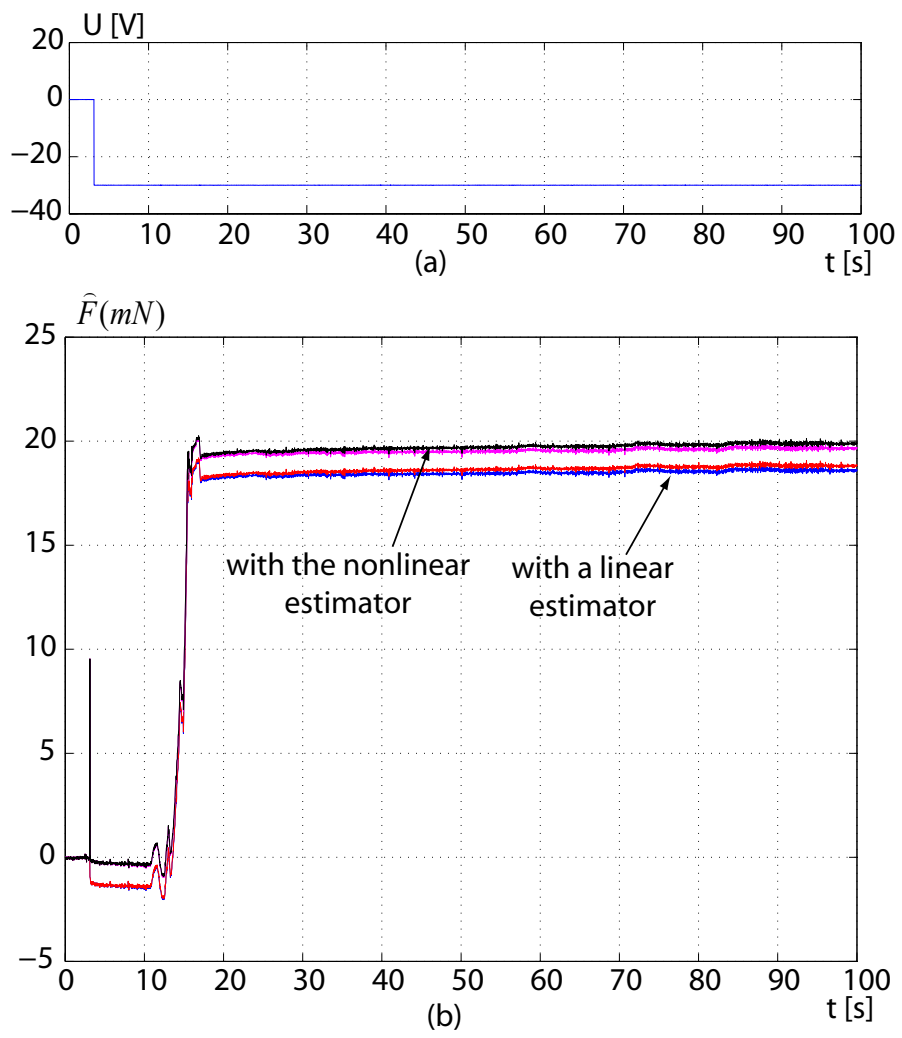

Fig. 15. Experimental results of a force estimation.

the manipulated part. The accuracies of the corresponding forces are milli- or submillinewton. However, the limit of the existing technology is not yet able to process sensors with such accuracies and with sizes appropriate to the sizes of the microsystems. Hence, the development of force estimators has a great interest. In piezoelectric cantilevers, the classical linear force estimator has its limits when the working voltages become large. Nonlinearities, i.e. the hysteresis and the creep, must be taken into account. We proposed a nonlinear force estimator for piezoelectric cantilevers in this paper. To perform that, a complete model of the system has been developped. Experiments of force estimation were performed and compared with the results that a linear estimator would give. Their comparison has pointed substantial accuracies given by the proposed method.

\section{ACKNOWLEDGMENT}

This work is partially supported by the European Project EUPASS (http://www.eupass.org/).

\section{REFERENCES}

[1] R.S. Fearing, "Survey of sticking effect for micro parts handling" Proceeding of International Conference on Intelligent Robots and Systems, IROS'95, Vol.2, pp-212-227, Pittsburgh, PA, USA 1995.

[2] Y. Haddab, N. Chaillet, A. Bourjault, 'A microgripper using smart piezoelectric actuators', Proc. of the 2000 IEEE/RSJ International Conference on Intelligent Robots and Systems, IROS'2000, sur CD ROM, 6 pages, 31 octobre-5 novembre 2000, Kagawa University, Takamatsu, Japon.
3] J. Agnus, P. De Lit, C. Clévy, N. Chaillet, 'Description and performances of a four-degrees-of-freedom piezoelectric gripper', Proc. of the International Symposium on Assembly and Task Planning, ISATP'2003, -From the assembly and disassembly of manufactured products to the design and manufacturing of micromachines-, pp. 66-71, 10-11 juillet 2003, Besançon, France.

[4] Yassine Haddab, 'Conception et réalisation d'un système de micromanipulation contrôlé en effort et en position pour la manipulation d'objets de taille micrométrique', $\mathrm{PhD}$ Thesis, Franche-Comté University, FRANCE, 2000.

[5] Jinghua Zhong, Stefan Seelecke, Ralph C. Smith and Christof Büskens, 'Optimal control of piezoceramic actuators'.

[6] Y. Haddab, N. Chaillet and A. Bourjault, 'A microgripper using smart piezoelectric actuators' Proc. of the 2000 IEEE/RSJ International Conference on Intelligent Robots and Systems, IROS'2000, october-november 2000, Kagawa University, Takamatsu, Japan.

[7] I.D. Mayergoyz, 'Mathematical models of hysteresis', Springer Verlag, 1991.

[8] Micky Rakotondrabe, 'Développement et commande modulaire d'une station de microassemblage', $\mathrm{PhD}$ thesis, Université de Franche-Comté Besançon, 2006.

[9] R. Bouc, 'Forced vibration of mechanical systems with hysteresis', Proc. of the 4th Conference on Nonlinear Oscillations, Prague, Chechoslovakia, 1967.

[10] Y. K. Wen, 'Method for random vibration of hysteretic systems', J. Enr. Mech., 102:249-263, 1976.

11] M. Jouaneh and H. Tian, 'Accuracy enhancement of a piezoelectric actuators with hysteresis', Japan/USA Symp. Flexible Automation, ASME, 1:631-637, 1992.

[12] T. S. Low and W. Guo, 'Modeling of a three-layer piezoelectric bimorph beam with hysteresis', Journal of Microelectromechanical Systems, 4(4):230-237, December, 1995.

[13] D. Croft, G. Shed and S. Devasia, 'Creep, hysteresis and vibration compensation for piezoactuators: atomic force microscope application', Journal of Dynamic System, Measurement and Control, ASME, 123:35-43, 2001.

[14] J. L. Pons, 'Emerging actuator technoologies: a micromechatronic approach', Wiley, (ISBN 0-470-09197-5), 2005. 